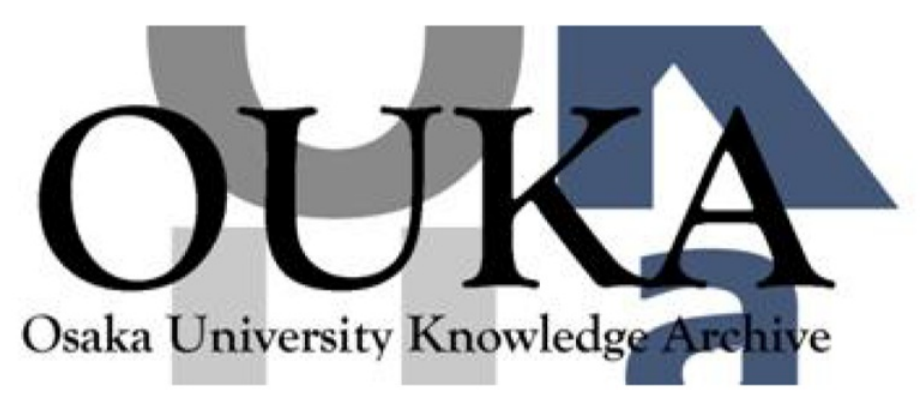

\begin{tabular}{|c|c|}
\hline Title & $\begin{array}{l}\text { Contactless mode-selective resonance ultrasound } \\
\text { spectroscopy : Electromagnet ic acoust ic } \\
\text { resonance }\end{array}$ \\
\hline Author(s) & $\begin{array}{l}\text { Ogi, Hirotsugu; Ledbetter, Hassel; Kim, Sudook } \\
\text { et al. }\end{array}$ \\
\hline Citation & $\begin{array}{l}\text { Journal of the Acoustical Society of America. } \\
106(2) \text { p. } 660-\text { p. } 665\end{array}$ \\
\hline Issue Date & 1999-08 \\
\hline oaire:version & AM \\
\hline URL & https://hdl. handle. net/11094/84152 \\
\hline rights & $\begin{array}{l}\text { Copyright } 1999 \text { Acoustical Society of America. } \\
\text { This article may be downloaded for personal use } \\
\text { only. Any other use requires prior permission } \\
\text { of the author and the Acoustical Society of } \\
\text { America. }\end{array}$ \\
\hline Note & \\
\hline
\end{tabular}

Osaka University Knowledge Archive : OUKA

https://ir. Library. osaka-u. ac. jp/

Osaka University 


\title{
Contactless mode-selective resonance ultrasound spectroscopy: Electromagnetic acoustic resonance
}

\author{
Hirotsugu Ogi \\ Graduate School of Engineering Science, Osaka University, Machikaneyama 1-3, Toyonaka, \\ Osaka 560-8531, Japan and Materials Science and Engineering Laboratory, NIST, Boulder, \\ Colorado 80303 \\ Hassel Ledbetter and Sudook Kim \\ Materials Science and Engineering Laboratory, NIST, Boulder, Colorado 80303 \\ Masahiko Hirao \\ Graduate School of Engineering Science, Osaka University, Machikaneyama 1-3, Toyonaka, \\ Osaka 560-8531, Japan
}

(Received 15 December 1998; accepted for publication 12 May 1999)

\begin{abstract}
A noncontacting resonant-ultrasound-spectroscopy (RUS) method for measuring elastic constants and internal friction of conducting materials is described, and applied to monocrystalline copper. This method is called electromagnetic acoustic resonance (EMAR). Contactless acoustic coupling is achieved by energy transduction between the electromagnetic field and the ultrasonic vibrations. A solenoidal coil and static magnetic field induce Lorentz forces on specimen surfaces without using a coupling agent. By changing the field direction, a particular set of vibration modes can be selectively excited and detected, an advantage in identifying the vibration modes of the observed resonance peaks. Contactless coupling allows the measure of intrinsic internal friction free from energy loss associated with contact coupling. The elastic constants and internal friction measured by EMAR are compared with those by the usual RUS method for a rectangular-parallelepiped copper monocrystal. Both methods yielded the same elastic constants despite fewer resonant peaks in the EMAR case. The two methods gave essentially the same shear-mode internal friction, but the RUS method gave higher volume-mode internal friction. () 1999 Acoustical Society of America. [S0001-4966(99)05208-X]
\end{abstract}

PACS numbers: 43.35.Cg, 43.35.Yb [HEB]

\section{INTRODUCTION}

Resonant ultrasound spectroscopy (RUS) is recognized as a useful method to determine elastic constants of solids, even for a crystal of lower symmetry. The usual RUS configuration uses a sphere, cylinder, or rectangularparallelepiped specimen, whose dimensions are typically 10 to $1 \mathrm{~mm}$, and two sandwiching transducers that touch the specimen at opposite corners. One transducer generates a continuous wave $(\mathrm{cw})$ oscillation and the other detects ultrasonic oscillation. Sweeping through frequency, one obtains the frequency response of the received amplitude, which consists of a spectrum of resonance peaks. The resonance frequencies are then used in an inverse calculation to find the complete set of elastic constants. Good overviews of RUS were given by Maynard ${ }^{1}$ and Migliori and Sarrao. ${ }^{2}$

To make full use of the attractive feature of RUS that one can obtain all independent elastic constants with a single frequency scan on a single specimen, many efforts have been made both for measurement development and numerical calculation of the resonance frequencies. Concerning technical study and application, Migliori and his co-workers ${ }^{3}$ made large contributions. They developed instrumentation for acquiring the resonance spectrum of very small specimens $\left(\sim 0.001 \mathrm{~cm}^{3}\right)$ as a function of temperature $(20-400 \mathrm{~K})$. Kuokkala and Schwarz ${ }^{4}$ used a deposited nickel film for noncontact ultrasonic transduction and measured internal friction of an $\mathrm{Ni}_{80} \mathrm{P}_{20}$ alloy up to $520 \mathrm{~K}$. Ledbetter et al. ${ }^{5}$ applied
RUS to measure elastic constants of a boron-aluminum fiber-reinforced composite. More recently, Tanaka et al. ${ }^{6-8}$ measured elastic constants of monocrystals of intermetallic compounds, including $\gamma$-TiAl. Isaak et al. ${ }^{9}$ used it to measure the shear-modulus pressure derivative of fused silica and discussed the effect of mass density of pressurizing gases. Concerning numerical calculation, Holland ${ }^{10}$ used a Fourier series to approximate deformation in a cubic specimen. Demarest ${ }^{11}$ used Legendre polynomials and obtained a good solution with a smaller number of harmonic functions. Ohno $^{12}$ also used Legendre functions and established an effective method for obtaining free-vibration resonant frequencies.

A key to the successful determination of elastic constants is the correct correspondence between the observed and calculated resonance frequencies. Because the identification of the observed resonance modes is not straightforward, one needs to know beforehand a set of elastic constants near real values. Otherwise, the inversion fails to converge or converges to a false minimum. This forces us to supplement RUS with other methods such as a pulse-echo or rod resonance. To reduce these troubles, several efforts have been made for resonance-peak mode identification. Ohno ${ }^{12}$ used different rates of changes of resonant frequencies by varying the specimen size. Maynard ${ }^{13}$ switched assignments of frequencies during the iteration calculation for finding the best fit. Migliori et $a l^{3}{ }^{3}$ changed the sample orientation relative to 


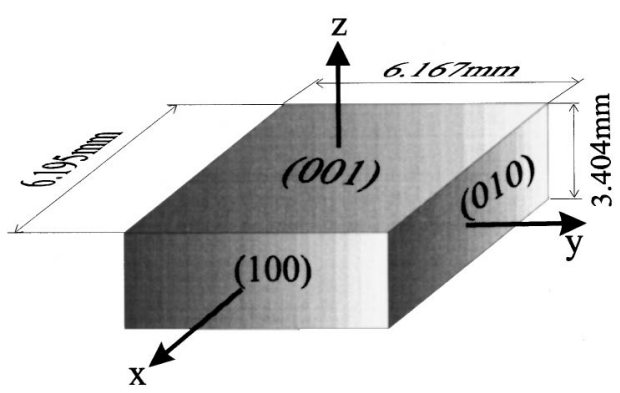

FIG. 1. Rectangular-parallelepiped specimen of copper monocrystal (6.159 $\left.\times 6.167 \times 3.404 \mathrm{~mm}^{3}\right)$.

the transducers and monitored the signal amplitude. Okamoto et al. ${ }^{14}$ used multiple detectors attached to different points on the surface to evaluate the vibration symmetry using the multiple outputs. These methods are laborious and sometimes inapplicable for a solid having larger internal friction because broad resonant peaks usually overlap one another. The best way to identify the mode is to independently produce only one group of vibrations, filtering others out. No one reported a method realizing this goal for RUS method.

In the present study, we present a new noncontacting RUS method to selectively excite and detect only one group of vibrations; electromagnetic acoustic resonance (EMAR). ${ }^{15-19}$ A specimen is inserted in a solenoidal coil where a uniform static magnetic field is applied. By driving the coil with a high-power rf burst, electromagnetic body forces occur directly on the specimen surfaces. The same coil works as a receiver. Because the direction and symmetry of the body forces can be easily controlled by changing the geometrical configuration of the static field and the coil, we can generate and detect only a selected subgroup of resonances. The acoustically contactless coupling has an especially important advantage in measuring internal friction. Little energy loss to transducers occurs, which helps us measure intrinsic internal friction. Another outstanding aspect of EMAR is its contactless feasibility at elevated temperatures. The authors ${ }^{19}$ made a noncontact monitoring of internal friction evolution in an $\mathrm{SiC} / \mathrm{Ti}$ composite up to $1000 \mathrm{~K}$. The present study demonstrates the usefulness of EMAR to determine elastic constants and internal friction with a rectangular-parallelepiped copper monocrystal.

\section{MODE-SELECTION METHOD}

Figure 1 shows the shape and size of the copper monocrystal used in the present study. The $x, y$, and $z$ axes are defined as shown. Archimedes-method mass density was $8.94 \mathrm{~g} / \mathrm{cm}^{3}$. The dimension fluctuation was within $0.3 \%$. Measurements were made at ambient temperature.

Figure 2 shows a typical measurement setup. The specimen was inserted into a thin plastic sheet in a solenoidal coil fixed between two permanent-magnet blocks. The solenoidal coil is loose and the specimen is unconstrained. Mechanical specimen-plastic coupling is weak because of no applied force, just specimen mass, and because of large acousticimpedance mismatch. Thus, this situation can be considered as acoustically noncontacting. The permanent magnets provide the static magnetic field needed for Lorentz-force cou-

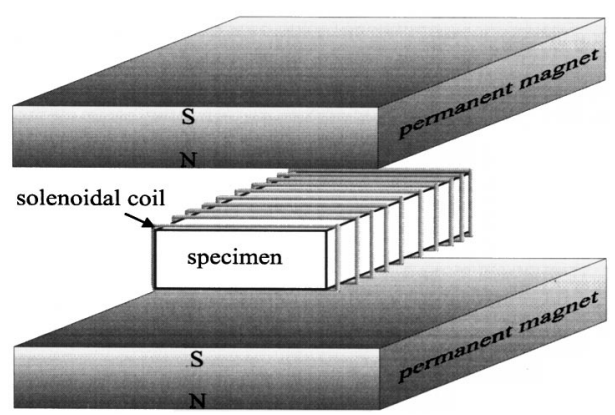

FIG. 2. Typical setup for electromagnetic-acoustic transduction.

pling. The magnetic field was $0.15 \mathrm{~T}$. Driving the coil with a high-power rf burst causes eddy currents on the surfaces, which interact with the magnetic field and generate Lorentz forces. These forces are the sources of the ultrasonic vibration. The reverse mechanism works in the receiving process. Details of the electromagnetic coupling into the acoustic wave can be found elsewhere. ${ }^{17-20}$

We measured the three configurations shown in Fig. 3: magnetic field parallel to $x$ axis and the resultant Lorentz forces normal to $x$ axis (case I); magnetic field parallel to $z$ axis and Lorentz forces parallel to $x$ axis (case II); and magnetic field parallel to $z$ axis and Lorentz forces parallel to $y$ axis (case III). Considering $x$-, $y$-, and $z$-components of displacement on the surfaces, $u, \mathrm{v}$, and $w$, respectively, we notice that in case I $w$ on $x-y$ faces can be detected by the reversed-Lorentz-force mechanism only when it is an odd function about $z$ and an even function about $x$ and $y$, and also $\mathrm{V}$ on $z-x$ faces can be detected only when it is an odd function about $y$ and an even function about $x$ and $z$. Similarly, in case II and case III, the Lorentz-force mechanism

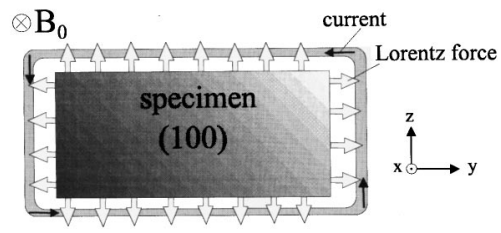

(a) Case I
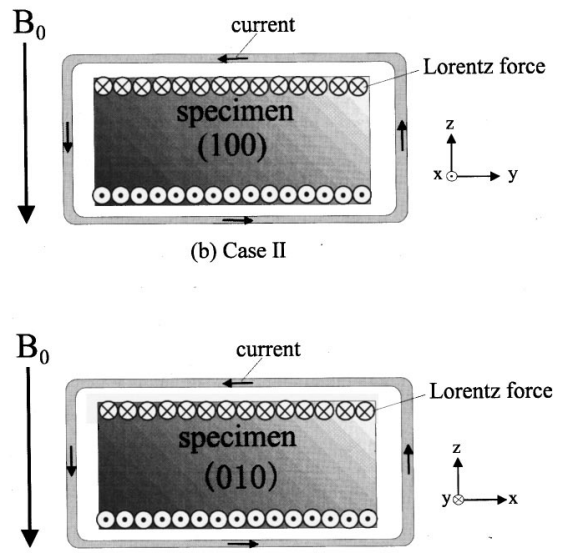

(c) Case III

FIG. 3. Three configurations of the coil and static field for generating (a) OD group, (b) OY group, and (c) OX group. 

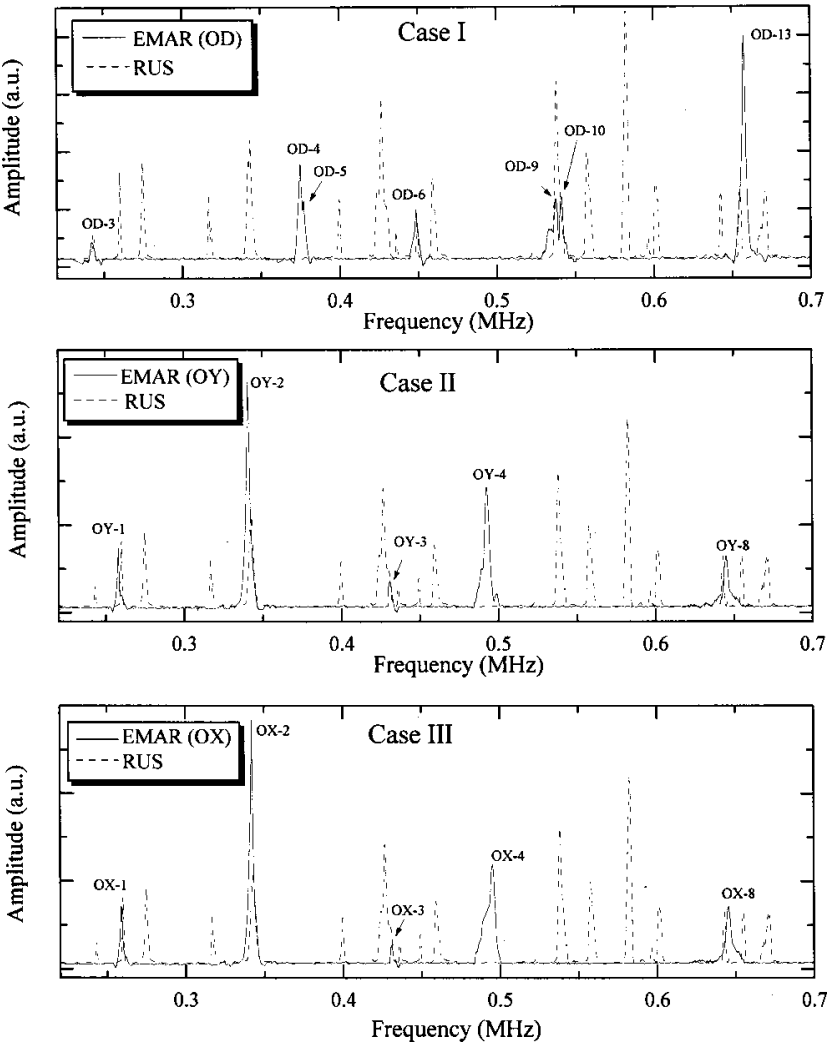

FIG. 4. Resonance spectrum for the three cases. RUS results are shown as a broken line.

works responding to $u$ and $w$ on $x-y$ faces, respectively. To detect them with the same coil, they must be odd functions about $z$ and even functions about $x$ and $y$. For a rectangularparallelepiped crystal with symmetry orthorhombic or higher, the vibration modes fall into eight groups, depending on the deformation symmetry. ${ }^{11,12}$ Among them, only the OD group (breathing vibration) satisfies the condition of the deformation symmetry of case I. Similar analysis shows that only the OY group (torsional vibration about the $y$-axis) can be detected in case II, and the OX group (torsional vibration about the $x$-axis) in case III. Thus, we can easily select the vibration group by changing the configuration of the measurement setup.

\section{THE ELASTIC CONSTANTS}

We show the resonance spectra in Fig. 4 for the three cases. For comparison, we made the usual RUS measurement using the same specimen. The detailed configuration of the RUS measurement in the present study is found in Ref. 5. The RUS result is shown in Fig. 4 with a dashed line, which demonstrates detection of many groups of vibration other than the above three. Different EMAR configurations led to different spectra, as expected. We determined the resonance frequencies by fitting the Lorentzian function to a peak and calculating its center. Although the number of the resonant peaks observed with EMAR is fewer than that with RUS, the determination of a set of elastic constants is straightforward. This is because mode identification is much easier in EMAR. For example, the OX-2 and OY-2 resonances occur at close frequencies, and they overlap in RUS measurement as shown

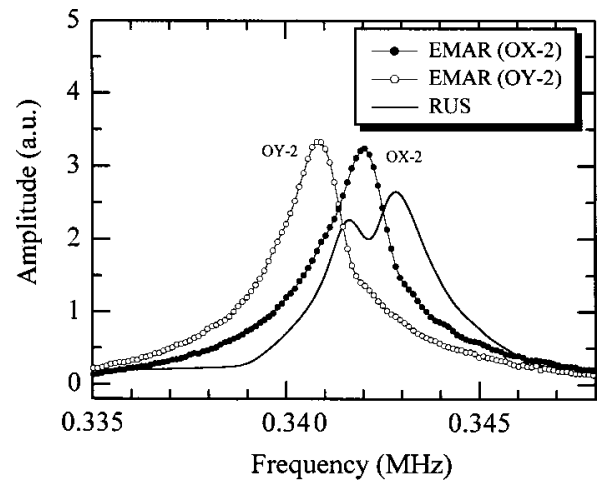

FIG. 5. Resonance spectra measured by EMAR and RUS.

in Fig. 5. However, because EMAR can select either of them, the distinction is easy, giving no ambiguity. We made the inverse calculation developed in previous studies, ${ }^{5,12}$ using the EMAR resonance frequencies, and we obtained the three cubic-symmetry elastic constants. Measured and calculated resonance frequencies are shown in Table I. (Equipment limitations prevented our detecting the lower-frequency modes OD-1 and OD-2.) Measured and calculated frequencies agreed within $0.4 \%$, which is comparable with the fractional error of the specimen dimensions. This iterative calculation needs a first guess of elastic constants. We started the iteration with several sets of elastic constants covering a wide range $\left(C_{11}: 100-230 \mathrm{GPa}, C_{12}: 70-150 \mathrm{GPa}\right.$, $C_{44}: 50-150 \mathrm{GPa}$ ) and we obtained the same results. We also deduced the elastic constants with the RUS frequencies using a larger number of resonances. In this case, to get correct convergence, we needed better guesses $\left(C_{11}=168 \mathrm{GPa}, C_{12}\right.$ $\left.=120 \mathrm{GPa}, C_{44}=74 \mathrm{GPa}\right)$. The measured and calculated frequencies also showed a difference of $0.4 \%$.

Table II shows the elastic constants deduced from the

TABLE I. Measured $\left(f_{\text {meas }}\right)$ and calculated $\left(f_{\text {calc }}\right)$ resonance frequencies for EMAR case.

\begin{tabular}{cccc}
\hline \hline Mode & $f_{\text {meas }}(\mathrm{MHz})$ & $f_{\text {calc }}(\mathrm{MHz})$ & Difference $(\%)$ \\
\hline OD-1 & $\ldots$ & 0.183706 & $\ldots$ \\
OD-2 & $\ldots$ & 0.21136 & $\ldots$ \\
OD-3 & 0.242323 & 0.242549 & 0.09 \\
OD-4 & 0.374916 & 0.373805 & -0.3 \\
OD-5 & 0.375139 & 0.374833 & -0.08 \\
OD-6 & 0.448678 & 0.450268 & 0.35 \\
OD-7 & $\ldots$ & 0.472787 & $\ldots$ \\
OD-8 & $\ldots$ & 0.494272 & $\ldots$ \\
OD-9 & 0.535587 & 0.53339 & -0.41 \\
OD-10 & 0.542134 & 0.543198 & 0.2 \\
OD-11 & $\ldots$ & 0.567346 & $\ldots$ \\
OD-12 & $\ldots$ & 0.64316 & $\ldots$ \\
OD-13 & 0.657429 & 0.65774 & 0.05 \\
& & & \\
OX-1 & 0.258849 & 0.258944 & 0.04 \\
OX-2 & 0.341657 & 0.341765 & 0.03 \\
OX-3 & 0.429726 & 0.428519 & -0.28 \\
OX-4 & 0.492967 & 0.494452 & 0.3 \\
OY-1 & 0.257887 & 0.258572 & 0.27 \\
OY-2 & 0.340418 & 0.340449 & 0.01 \\
OY-3 & 0.430686 & 0.42946 & -0.28 \\
OY-4 & 0.49249 & 0.49367 & 0.24 \\
\hline \hline
\end{tabular}


TABLE II. Elastic constants and internal friction of a copper monocrystal determined by EMAR and RUS.

\begin{tabular}{lcccccc}
\hline \hline & \multicolumn{3}{c}{$C_{i j}(\mathrm{GPa})$} & & $Q_{i j}^{-1}\left(\times 10^{-3}\right)$ \\
\cline { 2 - 5 } & EMAR & RUS & Ref. 21 & Ref. 22 & EMAR & RUS \\
\hline$C_{11}$ & 167.0 & 168.7 & 169.7 & 168.4 & 1.14 & 1.74 \\
$C_{12}$ & 120.9 & 121.6 & 122.6 & 121.4 & 0.30 & 1.34 \\
$C_{44}$ & 74.64 & 75.46 & 74.49 & 75.40 & 2.59 & 2.46 \\
$B=\left(C_{11}+2 C_{12}\right) / 3$ & 136.2 & 137.3 & 137.3 & 137.1 & 0.64 & 1.50 \\
$C^{\prime}=\left(C_{11}-C_{12}\right) / 2$ & 23.05 & 23.52 & 23.35 & 23.50 & 3.34 & 2.80 \\
$C_{110,110}=\left(C_{11}+C_{12}+2 C_{44}\right) / 2$ & 218.6 & 220.6 & 220.6 & 220.3 & 1.40 & 1.87 \\
$C_{111,111}=\left(C_{11}+2 C_{12}+4 C_{44}\right) / 3$ & 235.7 & 237.9 & 237.6 & 237.7 & 1.47 & 1.90 \\
$C_{111, \text { arb }}=\left(C_{11}-C_{12}+C_{44}\right) / 3$ & 40.25 & 40.84 & 40.54 & 40.80 & 2.88 & 2.59 \\
$C_{L}$ & 199.6 & 201.6 & $\ldots$ & $\ldots$ & 1.34 & 1.84 \\
$G$ & 47.54 & 48.22 & $\ldots$ & $\ldots$ & 2.83 & 2.57 \\
$E$ & 127.8 & 129.5 & $\ldots$ & $\ldots$ & 2.60 & 2.46 \\
$\nu$ & 0.3437 & 0.3428 & $\ldots$ & $\ldots$ & $\ldots$ \\
\hline \hline
\end{tabular}

two methods and those obtained previously, ${ }^{21,22}$ including other useful elastic-constant combinations obtained from $C_{11}, C_{12}$, and $C_{44}$. Using Kröner's method, ${ }^{23}$ we calculated the usual isotropic-material averaged-over-direction elastic constants: shear modulus $G$, longitudinal modulus $C_{L}$, Young's modulus $E$, and Poisson ratio $\nu$. EMAR provided slightly smaller stiffness constants than those measured by the RUS method. This may be caused by the mechanical coupling of RUS, even if it is weak; that is, RUS does not achieve ideal free vibration. To obtain the resonance frequencies of free vibration with RUS, one should minimize the forces from the sandwiching piezoelectric transducers. Applied load partially constrains the sample deformation and increases the resonant frequencies (see Fig. 5), leading to larger elastic constants in general. ${ }^{24}$ We noticed this influence during measurements when the force was changed. However, since we did our best to minimize the force in the RUS measurement (it may be slightly larger than the sample mass), the influence on the elastic constants should be insignificant.

EMAR does not detect all vibration modes expected for a given configuration (see Fig. 3). For example, OD-11 and OD-12 were missing in case I. These absences occur because the solenoidal coil detects the integral displacement over the specimen faces. If this value is too small, EMAR fails to detect that mode. It would be most useful to develop a model that predicts vibration-mode amplitudes. Then, we could predict low-amplitude modes and further facilitate mode identification.

Another configuration between the coil and static field will make it possible to generate other vibration groups. For example, designing a solenoidal coil where the direction of the driving current is reversed at the center of the sample, one can change the deformation symmetry that can be detected by the coil. Considering that the axial direction of such a coil is along the $x$ axis and the static field is applied along the $x$ axis, displacement $w$ on $x-y$ faces can be detected only if it is an odd function about $z$, an even function about $y$, and an odd function about $x$. This is known as the EX mode ${ }^{12}$ (symmetric flexure vibration along the $x$ axis). In principle, one can select one of seven classes of vibration modes with the present method. Not excitable is the EV mode (asymmetric flexure mode).

\section{THE INTERNAL FRICTION}

EMAR has the important advantage that no energy loss occurs from mechanical contact. Thus, it is ideal for measuring internal friction. The measurement is never affected by uncertain changes of mechanical-coupling conditions, which are accompanied by varying temperature and pressure (or stress). Thus, together with the applicability to in situ monitoring, intensive studies have been made using EMAR to detect the evolution of internal friction at elevated temperatures, ${ }^{18,19}$ during deformation, ${ }^{25}$ recovery, ${ }^{26}$ and fatigue. ${ }^{27}$

Here, we compare the internal friction measured by EMAR and RUS. In RUS measurements, internal friction is usually obtained from the resonance peak width: $Q^{-1}$ $=\Delta f / f_{r}$, where $\Delta f$ denotes the peak width at the half-power amplitude and $f_{r}$ resonance frequency. We determined $Q^{-1}$ by fitting a Lorentzian function to the resonance peak and calculating $\Delta f$. In EMAR, internal friction can be measured from the resonance ringdown. ${ }^{16}$ After an excitation with a burst signal at a resonance frequency, the reverberation exponentially decays with time. This ringdown curve provides the attenuation coefficient $\alpha$ by fitting an exponential function. Figure 6 shows an example of the measured ringdown curve and the fitted exponential function for the OX-2 mode. We show the same measurements on a logarithmic scale. Departure from the fitted curve at low-amplitude region is caused by the background noise. $Q^{-1}$ and $\alpha$ are related by

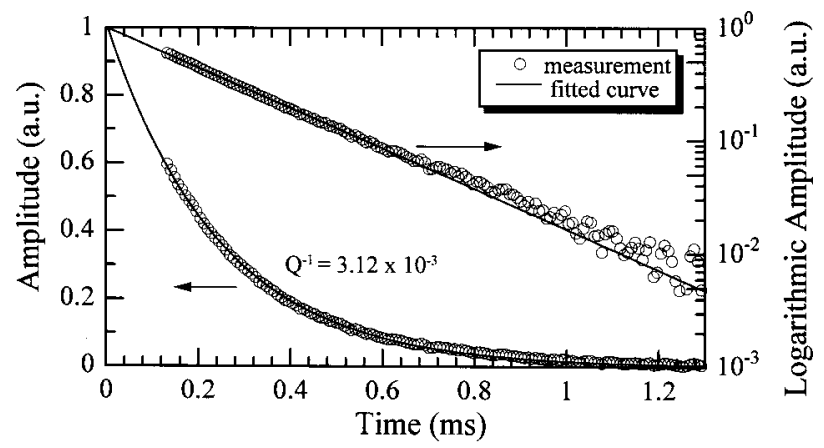

FIG. 6. Measured ringdown curve of OX-2 mode with EMAR. Solid line is a fitted exponential function. Logarithmic-scale amplitude for the same data is also shown. 
TABLE III. Internal friction measured by $\operatorname{EMAR}\left(Q_{\mathrm{EMAR}}^{-1}\right)$ and RUS $\left(Q_{\mathrm{RUS}}^{-1}\right)$.

\begin{tabular}{cccc}
\hline \hline Mode & $Q_{\text {EMAR }}^{-1}\left(\times 10^{-3}\right)$ & $Q_{\text {RUS }}^{-1}\left(\times 10^{-3}\right)$ & Difference $(\%)$ \\
\hline OD-1 & $\ldots$ & 2.63 & $\ldots$ \\
OD-2 & $\ldots$ & 1.36 & $\cdots$ \\
OD-3 & 2.73 & 3.52 & -29 \\
OD-4 & 4.46 & 2.68 & 39 \\
OD-5 & 4.33 & 2.80 & 35 \\
OD-6 & 2.10 & 3.00 & -43 \\
OD-7 & $\cdots$ & $\cdots$ & $\ldots$ \\
OD-8 & $\ldots$ & $\ldots$ & $\cdots$ \\
OD-9 & 3.01 & 3.04 & -1 \\
OD-10 & 1.44 & 1.51 & -5 \\
OD-11 & $\cdots$ & 3.38 & $\cdots$ \\
OD-12 & $\ldots$ & 1.71 & -12 \\
OD-13 & 1.55 & 1.74 & -8 \\
& & & -21 \\
OX-1 & 2.20 & 2.38 & 6 \\
OX-2 & 3.12 & 3.78 & $\cdots$ \\
OX-3 & 1.94 & 1.83 & -17 \\
OX-4 & 4.01 & $\cdots$ & -9 \\
OY-1 & 2.42 & 2.82 & $\cdots$ \\
OY-2 & 3.16 & 3.43 & \\
OY-3 & 2.03 & 2.06 & $\cdots$ \\
OY-4 & 3.87 & & \\
\hline \hline
\end{tabular}

$Q^{-1}=\alpha /\left(f_{r} \pi\right)$. In the EMAR case, $\Delta f / f_{r}$ should not be used for $Q^{-1}$ because at a slightly off-resonance frequency the burst wave cannot cancel the ringdown signal adequately, unlike the cw signal, resulting in a broader resonance peak width.

Table III compares $Q^{-1}$ measured by RUS and EMAR. For most modes, $Q^{-1}$ from RUS exceeded that from EMAR. OD-4 and OD-5 modes showed larger $Q^{-1}$ in the EMAR measurement. RUS $Q^{-1}$ values for these modes are not reliable because of the very low resonance intensity (see Fig. 4). Because we can calculate the contribution of each elastic constant to the resonant frequencies, we can also calculate the internal friction for each elastic constant; that is, the internal friction tensor $Q_{i j}^{-1} \cdot{ }^{28,29} Q_{i j}^{-1}$ is useful because one can calculate the internal friction of any oscillation mode. We show $Q_{i j}^{-1}$ thus calculated in Table II. Internal friction for the bulk modulus $B$ was considerably smaller. Because in monocrystal copper a large part of the internal friction is caused by dislocation damping, this observation is easily understood because of the lower mobility of dislocations during hydrostatic loading. The larger internal friction of shear

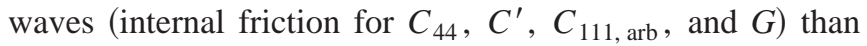
those of longitudinal waves $\left(C_{11}, C_{110,110}, C_{111,111}\right.$, and $\left.C_{L}\right)$ indicates that shear waves more effectively cause dislocation vibration about their pinning points than do longitudinal waves. This is supported by a simultaneous measurement of shear wave and longitudinal wave internal friction on deforming polycrystalline copper. ${ }^{25}$ The ratio of internal friction for longitudinal modulus $\left(C_{L}\right)$ and shear modulus $(G)$ was 2.1 for EMAR. The ratio for RUS was 1.4 , which is comparable with a ratio 1.6 found by Ledbetter and co-workers ${ }^{28}$ in studying polycrystalline copper using RUS. Comparing EMAR and RUS, we find a larger RUS $Q_{i j}^{-1}$ for longitudinal waves and, especially, for the bulk modulus; the
$Q_{i j}^{-1}$ for shear waves were comparable. This indicates that vibration modes accompanying volume change cause energy losses into the sandwiching transducers in an RUS measurement, even when the loading force is minimized.

The only possible extra energy loss in EMAR is eddycurrent loss in the specimen. Ultrasonic vibration in a uniform magnetic field generates eddy currents within the specimen. This loss is estimated by ${ }^{16}$

$$
Q_{\mathrm{ed}}^{-1}=\frac{\sigma B_{0}^{2}}{\omega \rho} .
$$

Here, $\sigma$ denotes electric conductivity, $B_{0}$ flux density of the static field, $\omega$ angular frequency, and $\rho$ mass density. Taking $\sigma=6 \times 10^{7} \mathrm{~S} / \mathrm{m}, \quad \rho=8.94 \mathrm{~g} / \mathrm{cm}^{3}, \quad f=0.2 \mathrm{MHz}$, and $B_{0}$ $=0.15 \mathrm{~T}$, we have $Q_{\text {ed }}^{-1}=1.2 \times 10^{-4}$, which is small compared with the measured $Q^{-1}$. One may consider that $Q_{\text {ed }}^{-1}$ has to be compensated for a material of low $Q^{-1}$ and high conductivity. Fortunately, such a material shows good electromagnetic-acoustic transduction efficiency and we can lower the static magnetic field and thus also $Q_{\text {ed }}^{-1}$.

\section{CONCLUSIONS}

(1) Electromagnetic acoustic resonance (EMAR) works well in measuring elastic constants, even with a smaller number of resonant peaks than in RUS. By changing the geometrical configuration of the coil and static field, one can selectively generate and detect a particular group of vibration modes. This helps identify vibration modes for the elastic-constant determination.

(2) The inverse calculation using the mode-identified resonant frequencies with EMAR provided one set of elastic constants, being independent of the initial guessed values for iteration. The RUS method can be extremely sensitive to the first guess.

(3) Elastic constants measured by EMAR were generally smaller than those by RUS with contacting transducers by approximately $1 \%$.

(4) Except for the pure-shear modes, internal friction measured by EMAR was lower than that by RUS for all useful elastic constant combinations. The difference was substantial for the breathing modes because the RUS method involves energy loss into the transducers, even for minimum coupling load.

1J. Maynard, “'Resonant ultrasound spectroscopy,', Phys. Today 49, 26 (1996).

${ }^{2}$ A. Migliori and J. Sarrao, Resonant Ultrasound Spectroscopy (Wiley, New York, 1997).

${ }^{3}$ A. Migliori, J. Sarrao, M. W. Visscher, T. Bell, M. Lei, Z. Fisk, and R. Leisure, "Resonant ultrasound spectroscopic technique for measurement of the elastic moduli of solids,', Physica B 183, 1 (1993).

${ }^{4}$ V.-T. Kuokkala and R. B. Schwarz, "The use of magnetostrictive film transducers in the measurement of elastic moduli and ultrasonic attenuation of solids,' Rev. Sci. Instrum. 63, 3136 (1992).

${ }^{5}$ H. Ledbetter, C. Fortunko, and P. Heyliger, " Orthotropic elastic constants of a boron-aluminum fiber-reinforced composite: An acoustic-resonancespectroscopy study,', J. Appl. Phys. 78, 1542 (1995).

${ }^{6}$ K. Tanaka, T. Ichitsubo, H. Inui, M. Yamaguchi, and M. Koiwa, "Singlecrystal elastic constants of $\gamma$-TiAl,', Philos. Mag. A 73, 71 (1996).

${ }^{7}$ K. Tanaka and M. Koiwa, "Single-crystal elastic constants of intermetallic compounds,', Intermetallics 4, 29 (1996).

${ }^{8}$ K. Tanaka, K. Okamoto, H. Inui, Y. Minonishi, M. Yamaguchi, and M. 
Koiwa, "Elastic constants and their temperature dependence for the intermetallic compound $\mathrm{Ti}_{3} \mathrm{Al}$," Philos. Mag. A 73, 1475 (1996).

${ }^{9}$ D. Isaak, J. Carnes, O. Anderson, and H. Oda, "Elasticity of fused silica spheres under pressure using resonant ultrasound spectroscopy," J. Acoust. Soc. Am. 104, 2200 (1998).

${ }^{10} \mathrm{R}$. Holland, "Resonant properties of piezoelectric ceramic rectangular parallelepipeds," J. Acoust. Soc. Am. 43, 988 (1968).

${ }^{11} \mathrm{H}$. Demarest, Jr., "Cube-resonance method to determine the elastic constants of solids," J. Acoust. Soc. Am. 49, 768 (1971).

${ }^{12}$ I. Ohno, "Free vibration of a rectangular parallelepiped crystal and its application to determination of elastic constants of orthorhombic crystals," J. Phys. Earth 24, 355 (1976).

${ }^{13} \mathrm{~J}$. Maynard, "The use of piezoelectric film and ultrasound resonance to determine the complete elastic tensor in one measurement," J. Acoust. Soc. Am. 91, 1754 (1992)

${ }^{14} \mathrm{~K}$. Okamoto, K. Tanaka, and M. Koiwa, Proceedings of the Second Pacific Rim International Conference on Advanced Materials and Processing, edited by K. S. Shin, J. K. Yoon, and S. J. Kim (Korean Institute of Metals and Materials, 1995), p. 1153.

${ }^{15}$ M. Hirao, H. Ogi, and H. Fukuoka, "'Resonance EMAT system for acoustoelastic stress evaluation in sheet metals," Rev. Sci. Instrum. 64, 3198 (1993).

${ }^{16} \mathrm{H}$. Ogi, M. Hirao, and T. Honda, "Ultrasonic attenuation and grain size evaluation using electromagnetic acoustic resonance," J. Acoust. Soc. Am. 98, 458 (1995).

${ }^{17}$ M. Hirao and H. Ogi, "Electromagnetic acoustic resonance and materials characterization," Ultrasonics 35, 413 (1997).
${ }^{18} \mathrm{~W}$. Johnson, "Ultrasonic damping in pure aluminum at elevated temperature," J. Appl. Phys. 83, 2462 (1998).

${ }^{19}$ H. Ogi, G. Shimoike, M. Hirao, K. Takashima, H. Ohtani, and H. Ledbetter, Review of Progress in QNDE, Vol. 18, edited by D. O. Thompson and D. E. Chimenti (Plenum, New York, 1999), p. 1337.

${ }^{20} \mathrm{H}$. Ogi, "Field dependence of coupling efficiency between electromagnetic field and ultrasonic bulk waves," J. Appl. Phys. 82, 3940 (1997).

${ }^{21} \mathrm{H}$. Ledbetter and S. Kim, Personal communication, unpublished.

${ }^{22}$ R. Hearmon, "Elastic constants of anisotropic materials-II," Adv. Phys. 5, 323 (1956).

${ }^{23}$ E. Kröner, "Self-consistent scheme and graded disorder in polycrystal elasticity,' J. Phys. F 8, 2261 (1978).

${ }^{24}$ Y. Sumino, I. Ohno, T. Goto, and M. Kumazawa, "Measurement of elastic constants and internal friction in single-crystal $\mathrm{MgO}$ by rectangular parallelepiped resonance," J. Phys. Earth 24, 263 (1976).

${ }^{25}$ H. Ogi, N. Suzuki, and M. Hirao, "Noncontact ultrasonic spectroscopy on deforming polycrystalline copper; dislocation damping and acoustoelasticity," Metall. Mater. Trans. A 29, 2987 (1998).

${ }^{26} \mathrm{H}$. Ogi, A. Tsujimoto, M. Hirao, and H. Ledbetter (unpublished).

${ }^{27}$ H. Ogi, M. Hirao, and K. Minoura, "Noncontact measurement of ultrasonic attenuation during rotating fatigue test of steel," J. Appl. Phys. 81, 3677 (1997)

${ }^{28}$ H. Ledbetter, C. Fortunko, and P. Heyliger, "Elastic constants and internal friction of polycrystalline copper," J. Mater. Res. 10, 1352 (1995).

${ }^{29} \mathrm{P}$. Heyliger and H. Ledbetter, in Mechanical Spectroscopy, edited by L. Magalas (Elsevier, Amsterdam, 1999) (unpublished). 